\title{
Prediction of Lymph Node Metastasis by Tumor Dimension Versus Tumor Biological Properties in Head and Neck Squamous Cell Carcinomas
}

\author{
Jeon Yeob Jang, $\mathrm{MD}, \mathrm{PhD}^{1}$ \\ Min Ji Kim, PhD² \\ Gwanghui Ryu, MD³ \\ Nayeon Choi, MD ${ }^{3}$ \\ Young-Hyeh Ko, MD, PhD ${ }^{4}$ \\ Han-Sin Jeong, MD, PhD 3
}

\begin{abstract}
Purpose
Lymph node metastasis (LNM) is a strong prognostic factor in many solid cancers, including head and neck squamous cell carcinomas (HNSCC), and LNM can be dependent upon primary tumor biology, as well as tumor dimension. Here, we investigate the relative risk of LNM in accordance to tumor dimension and biology in HNSCC subsites.
\end{abstract}

\section{Materials and Methods}

Medical data of 295 HNSCC patients who had undergone the initial curative surgery (oral tongue 174 , oropharynx 75 , hypopharynx 46 ) were analyzed to identify the significant predictive factor for LNM. Tumor volume and thickness were set as tumor dimensional variables, and biological variables included lymphovascular, perineural invasion, and tumor differentiation. Statistical analyses were conducted to assess the predictability of LNM from variables, and subgroup analyses according to the tumor subsites. In addition, we evaluated the impacts of tumor dimension and biological variables on the treatment outcomes and survival in HNSCC subsites.

\section{Results}

The overall tumor dimension and biological variables had a similar impact on the prediction of LNM in HNSCC (area under the curve, 0.7682 and 0.7717 ). The prediction sensitivity of LNM in oral tongue cancer was mainly dependent on tumor dimension, while LNM in oroand hypo-pharynx cancers was more influenced by biological factors. Survival analyses also confirmed that biological factor was more powerful in estimating disease-free survival of hypopharyngeal cancer patients, while tumor dimension was more significant in that of oral cancer patients.

\section{Conclusion}

Tumor dimension and biology have a significant, tumor subsite-dependent impact on the occurrence of LNM and disease-free survival in HNSCC.

\section{Key words}

Lymphatic metastasis, Head and neck neoplasms, Tumor burden, Tumor biological markers, Neoplasm metastasis

\section{Introduction}

Regional lymph node metastasis (LNM) is a well-known prognostic indicator in many types of solid cancer, including head and neck squamous cell carcinomas (HNSCC) [1]. LNM negatively influences the overall survival and increases the likelihood of distant metastases [2]. In HNSCC, the correlation between LNM and distant metastases is quite strong, especially with the presence of LNM in the neck being one of the strongest prognostic factors [2,3]. Moreover, the presence of LNM, number and size of involved lymph nodes, and nodal characteristics, including extra-capsular spread, significantly influence regional recurrence, distant metastases, and 
even overall survival [4,5]. Accurate diagnosis and therapeutic control of LNM is pivotal in the management of HNSCC [6].

The accepted treatment protocols for patients with HNSCC with clinical LNM are surgical ablation or chemoradiation therapy [6]. However, even in a clinically negative neck, surgical data from prophylactic neck dissection have demonstrated high rates of occult LNM for up to $34 \%$ of the patients in the majority of HNSCC [7]. Recently, several clinical and pathological features of primary tumors have been claimed to be predictive for occult metastases [8]. As the primary determinant of $\mathrm{T}$ classification, the maximum tumor diameter and invasion depth of primary tumors have been considered an important risk factor for regional LNM $[9,10]$. These data reflect the concept that regional LNM can be induced by time-dependent tumor burden or chronological age of primary tumors [11].

However, LNM from undetected or small primary lesions definitely exist in some cases, indicating that LNM is not always dependent upon the physical dimension of the primary tumors. Furthermore, a series of data presenting the significant correlation between lymphovascular invasion (LVI) and perineural invasion (PNI) with concomitant LNM suggest that the tumors with aggressive biological characteristics have higher risks of regional LNM $[12,13]$.

A recent report has confirmed that tumor dimension and biology have a similar impact on the presence of LNM in breast cancer [11]. Despite this body of knowledge, a few reports have linked these two phenotypes on LNM occurrence in HNSCC. Thus, this study was designed to identify the relative impact of tumor dimension and tumor biology on regional LNM in patients with HNSCC, particularly focusing on the subsites of the head and neck.

\section{Materials and Methods}

\section{Subject selection}

Medical information was extracted from a prospectively collected data in accordance to the standardized protocols in our institute for HNSCC, which included tumor size, thickness, and pathology details. Participants provided written informed consents. Among various subgroups of HNSCC, oral tongue, oropharynx (tonsil or base of tongue), and hypopharynx (pyriform sinus) cancers were included for analyses. Patients who had undergone surgery as the initial treatment between 2003 and 2012 were included. All pathology diagnoses were squamous cell carcinomas. Among them, the subjects were excluded from the analyses because of incomplete pathological information $(n=48)$ and followup duration of less than 2 years in case of no events $(n=55)$; the final number of study subjects was 295 . The baseline subject characteristics are summarized in Table 1. Of 295 HNSCC, oral tongue cancer was predominated $(n=174)$, followed by oropharynx and hypopharynx cancer. LNM was more prevalent in oropharynx and hypopharynx cancer compared to tongue cancer $(\mathrm{p}<0.0001$ and $\mathrm{p}=0.0028$, respectively). There was a significant correlation between $\mathrm{T}$ classification and LNM $(\mathrm{p}<0.0001)$. In patients with $\operatorname{LNM}(\mathrm{n}=153)$, distribution of N stage was N1 ( $=43), N 2(n=104)$, and N3 $(\mathrm{n}=6)$, respectively.

\section{Dimensional and biological variables of primary tumors}

The five major variables were selected as determinants of tumor dimension and biology. As tumor dimensional variables, we evaluated the tumor thickness and estimated tumor volume calculated from pathology specimen. The estimated tumor volume was calculated by the equation: $1 / 2 \times$ maximal diameter $\times$ minimal diameter $\times$ tumor thickness. As biological variables, we evaluated the presence of LVI, PNI, and differentiation status of primary tumors (=tumor grade). Patients with all available data for the five major variables were only included in the analyses.

To investigate the impact of tumor dimension and biology on disease recurrence/ systemic metastasis and survival, the survival analyses were conducted. The events of interest were defined as progression to recurrence or distant metastasis. Follow-up durations were defined as time gap between the completion of initial treatment, including adjuvant therapy, and the occurrence of events of interest. The mean follow-up duration was 32.5 months (range, 0 to 154 months). The events of interest within 1 month after completion of treatment were defined as a follow-up duration of 0 .

\section{Statistical analyses}

The baseline characteristics were analyzed for association of LNM, using a chi-square test for categorical variables and Wilcoxon rank sum tests for continuous variables. Post-hoc comparison of the association with primary tumor subsites and LNM was assessed by Fisher exact test using permutation method for multiple testing. The association between the selected variables and the presence of LNM with adjustment of primary tumor subsite was determined by a logistic regression analysis. To evaluate the predictive ability for five variables of tumor dimension and biology on the presence of LNM, the area under the curves (AUC) was calculated from receiver operating characteristics (ROC) curve analysis. The combined measures for tumor dimension and tumor biology were defined by a logistic regression model for two 
Table 1. Baseline characteristics of subjects and univariate analysis for lymph node metastasis

\begin{tabular}{|c|c|c|c|c|}
\hline Characteristic & Total $(n=295)$ & Node-negative ( $\mathrm{n}=142$ ) & Node-positive ( $n=153$ ) & p-value \\
\hline Age (yr) & $57(50-66)$ & $55(48-64)$ & $59(51-69)$ & 0.007 \\
\hline Sex (male:female) & $222: 73(75: 25)$ & $95: 47(67: 33)$ & $127: 26(83: 17)$ & 0.001 \\
\hline \multicolumn{5}{|l|}{ Tumor subsite } \\
\hline Oral cavity (tongue) & $174(59)$ & $108(37)$ & $66(22)$ & \multirow[t]{3}{*}{$<0.001$} \\
\hline Oropharynx & $75(25)$ & $18(6)$ & $57(19)$ & \\
\hline Hypopharynx & $46(16)$ & $16(5)$ & $30(10)$ & \\
\hline \multicolumn{5}{|l|}{ T-classification } \\
\hline $\mathrm{T} 1$ & $111(38)$ & $84(59)$ & $27(18)$ & \multirow[t]{4}{*}{$<0.001$} \\
\hline $\mathrm{T} 2$ & $111(38)$ & $32(23)$ & $79(52)$ & \\
\hline $\mathrm{T} 3$ & $33(11)$ & $13(9)$ & $20(13)$ & \\
\hline $\mathrm{T} 4$ & $40(14)$ & $13(9)$ & $27(18)$ & \\
\hline \multicolumn{5}{|l|}{ Tumor dimension variables } \\
\hline Tumor volume $\left(\mathrm{cm}^{3}\right)$ & $1.386(0.325-5.760)$ & $0.453(0.150-1.430)$ & $3.500(1.280-8.400)$ & $<0.001$ \\
\hline Tumor thickness (cm) & $0.8(0.4-1.6)$ & $0.5(0.3-1.0)$ & $1.2(0.7-1.8)$ & $<0.001$ \\
\hline \multicolumn{5}{|l|}{ Tumor biology variables } \\
\hline \multicolumn{5}{|l|}{ Lymphovascular invasion } \\
\hline Absent & $217(74)$ & $129(91)$ & $88(58)$ & \multirow[t]{2}{*}{$<0.001$} \\
\hline Present & $78(26)$ & $13(9)$ & $65(42)$ & \\
\hline \multicolumn{5}{|l|}{ Perineural invasion } \\
\hline Absent & $243(82)$ & $122(86)$ & $121(79)$ & \multirow[t]{2}{*}{0.124} \\
\hline Present & $52(18)$ & $20(14)$ & $32(21)$ & \\
\hline \multicolumn{5}{|l|}{ Tumor grade } \\
\hline Well-differentiated & $143(48)$ & $91(64)$ & $52(34)$ & \multirow[t]{3}{*}{$<0.001$} \\
\hline Moderately-differentiated & $130(44)$ & $44(31)$ & $86(56)$ & \\
\hline Poorly-differentiated & $22(7)$ & $7(5)$ & $15(10)$ & \\
\hline
\end{tabular}

Values are presented as median (25th-75th percentile) or number (\%). p-values are derived from Wilcoxon rank sum tests or chi-square tests depending on the type of variables.

and three variables. In order to assess the significance for the added predictive ability of the combined measure from the single variable, the integrated discrimination improvement (IDI) method was used. Subgroups analyses of LNM predictability, in accordance to the primary tumor subsites, were used. Univariate survival analyses of the possible clinical factors, tumor dimensional, and biological variables were conducted, using log-rank tests, and then Cox proportional hazards model was used to assess the association of the selected variables on disease-free and overall survival. All statistical analyses were executed using SAS ver. 9.3 (SAS Institute, Cary, NC). A p $<0.05$ was considered statistically significant. If needed, Bonferroni's correction was used for multiple testing, such as subgroup analysis.

\section{Results}

\section{Impact of variables of tumor dimension and biology on the occurrence of LNM}

Univariate analyses of five major variables regarding tumor dimension and biology revealed significant association with the presence of LNM, except PNI (Table 1). Because tumor subsite was significantly associated with the presence of LNM, we performed multivariate analyses for the variables of tumor dimension and biology adjusting to tumor subsite, which also showed a significant correlation of all these five variables with the occurrence of LNM (Table 2).

Next, to evaluate the predictability of LNM by variables of tumor dimension and biology, we performed ROC curve analysis. Both tumor volume and thickness could predict the presence of LNM with an AUC of 0.7546 and 0.7570, respectively (Fig. 1A). A combined measure of tumor dimension 
Table 2. Tumor dimension and biology for the occurrence of lymph node metastasis adjusting for tumor subsites

\begin{tabular}{lrrr} 
& \multicolumn{3}{c}{ Lymph node metastasis } \\
\cline { 2 - 4 } Variable & OR & $95 \%$ CI & p-value \\
Tumor dimension variables & & & \\
$\quad$ Tumor volume & 1.048 & $1.007-1.091$ & 0.022 \\
$\quad$ Tumor thickness & 3.328 & $2.207-5.017$ & $<0.001$ \\
Tumor biology variables & & & \\
$\quad$ Lymphovascular invasion & 7.530 & $3.797-14.932$ & $<0.001$ \\
$\quad$ Peri-neural invasion & 2.975 & $1.523-5.811$ & 0.001 \\
$\quad$ Tumor grade & & \multicolumn{2}{c}{} \\
$\quad$ MD/PD vs. WD & 2.191 & $1.286-3.735$ & 0.004 \\
\hline
\end{tabular}

The odds ratios (ORs) and p-values are derived from logistic regression model adjusted to the tumor subsites. $\mathrm{CI}$, confidence interval; $\mathrm{MD}$, moderately differentiated; $\mathrm{PD}$, poorly-differentiated; $\mathrm{WD}$, well-differentiated.

had increased AUC of 0.7682 and significantly increased the sensitivity without sacrificing specificity. Variables of LVI, PNI, and tumor grade could also predict LNM with AUC of $0.6666,0.5342$ and 0.6518 , respectively (Fig. 1B). The combined measure of tumor biology increased AUC of 0.7717, and significantly increased the sensitivity.

Combined measure of all five variables representing tumor dimension and biology strikingly increased the predictability of LNM with AUC of 0.8315 (Fig. 1C). Comparisons of predictive sensitivity between combined measures of tumor dimension and biology indicated no significant difference (IDI, 0.0223 ; $95 \%$ confidence interval, -0.0607 to $0.0954 ; \mathrm{p}>$ 0.999), suggesting that each combined measure of tumor dimension and biology had a similarly equivalent impact on the occurrence of LNM in HNSCC.

\section{Subsite difference in predictability for LNM from tumor dimension and biology variables}

These results led us to investigate the roles of tumor dimension and biology on each subsite of HNSCC. Interestingly, in oral tongue cancers, the predictive ability of tumor dimensional factors (AUC, 0.8403) was higher than biological factors (AUC, 0.7515) (Fig. 2A). A combined measure of all variables did not significantly increase the predictive sensitivity for LNM compared to tumor dimension ( $\mathrm{p}=0.076$ ), but was statistically significant in comparison with tumor biology ( $p<0.001)$, indicating that the predictive sensitivity of LNM in oral tongue cancer is mainly dependent on tumor dimensional factors.

On the other hand, in oropharynx cancers, the predictive ability for LNM was rather low in tumor dimensional factors (AUC, 0.7125$)$ in comparison to tumor biological factors (AUC, 0.8070) (Fig. 2B). A combined measure of all variables significantly increased the predictive sensitivity for LNM compared to tumor dimension ( $\mathrm{p}=0.006$ ), but had no statistical significance with respect to tumor biology $(\mathrm{p}=0.789)$, suggesting that the predictive sensitivity of LNM in oropharyngeal cancer is highly dependent on tumor biological factors. Similarly, in hypopharynx cancers, tumor dimensional factors had almost no predictive ability for LNM (AUC, 0.4354 ) and only biological factors had predictive ability for LNM (AUC, 0.7094) (Fig. 2C). A combined measure of all variables significantly increased the predictive sensitivity for LNM compared to tumor dimension (IDI=0.1391, $\mathrm{p}=0.040$ ), but had no statistical significance in comparison to tumor biology (IDI $=0.0040, p>0.999$ ). Thus, the impact of tumor dimension and biology was context-dependent in terms of tumor subsites in HNSCC; particularly in oro- and hypo-pharyngeal cancers, tumor progression to regional lymph nodes was influenced more by biological factors of primary tumor, rather than tumor dimension.

\section{Impact of tumor dimension and biology on disease out- comes and survival}

Previous studies have indicated a strong correlation between LNM in HNSCC and distant metastasis [2,3]. To evaluate whether the dimension or biology of the primary tumor affects the occurrence of distant metastasis or recurrence of loco-regional sites, 281 study patients were analyzed for disease-free survival. We excluded 14 patients from survival analyses due to incomplete medical and follow-up data among 295 initial subjects. Estimated disease-free survival rate of the total subjects was $74.9 \%$ at 2 years and $70.4 \%$ at 5 years.

First, we divided the study subjects into three subsite groups to identify the difference of relative impact of tumor dimensional or biological factors. Univariate analyses included age, sex, nodal status $(\mathrm{N})$, treatment modalities, and tumor dimensional or biological factors. T classification was significantly correlated with tumor dimensional variables; thus $\mathrm{T}$ classification was excluded from the subsequent analyses. Using potential factors significant in univariate analyses ( $\mathrm{p}<0.2)$, we conducted multivariate analyses, via a stepwise-selection method (Table 3).

In concordance to the previous findings, analyses using Cox proportional hazards model also confirmed the subsite differences of relative tumor dimensional or biological impacts. In oral cavity cancer, one of the tumor dimensional variables, tumor thickness, remained significant in a multivariate analysis (hazard ratio [HR], 4.518; $\mathrm{p}=0.016$ ); meanwhile, one of the biological variables, PNI, had a significant 

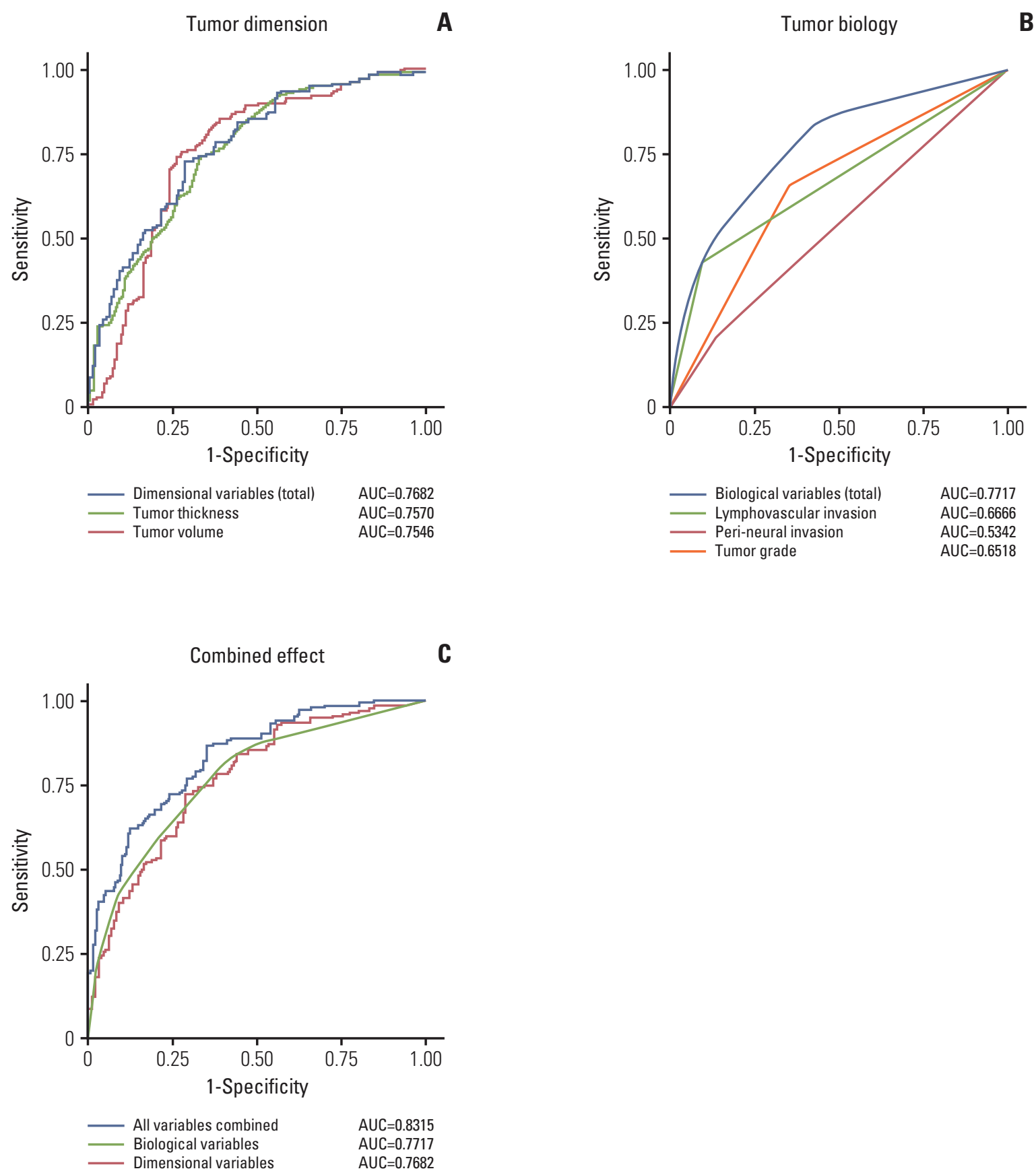

Fig. 1. Receiver operating characteristics (ROC) curve analyses to estimate predictability for lymph node metastasis by tumor dimension and biology variables. ROC curve from tumor dimensional variables (A), tumor biological variables (B), and combined measure of tumor dimensional and biological variables (C). AUC, area under the curves.

impact on disease-free survival in hypopharyngeal cancer (HR, 20.314; $\mathrm{p}=0.007$ ) (Table 3).

We further analyzed the impact of tumor dimension and biology on overall survival. Estimated overall survival rate was $78.5 \%$ at 2 years and $67.6 \%$ at 5 years. However, the results indicated that each variable of tumor dimension and tumor biology had no significant association with overall survival in our study patients (data not shown). 

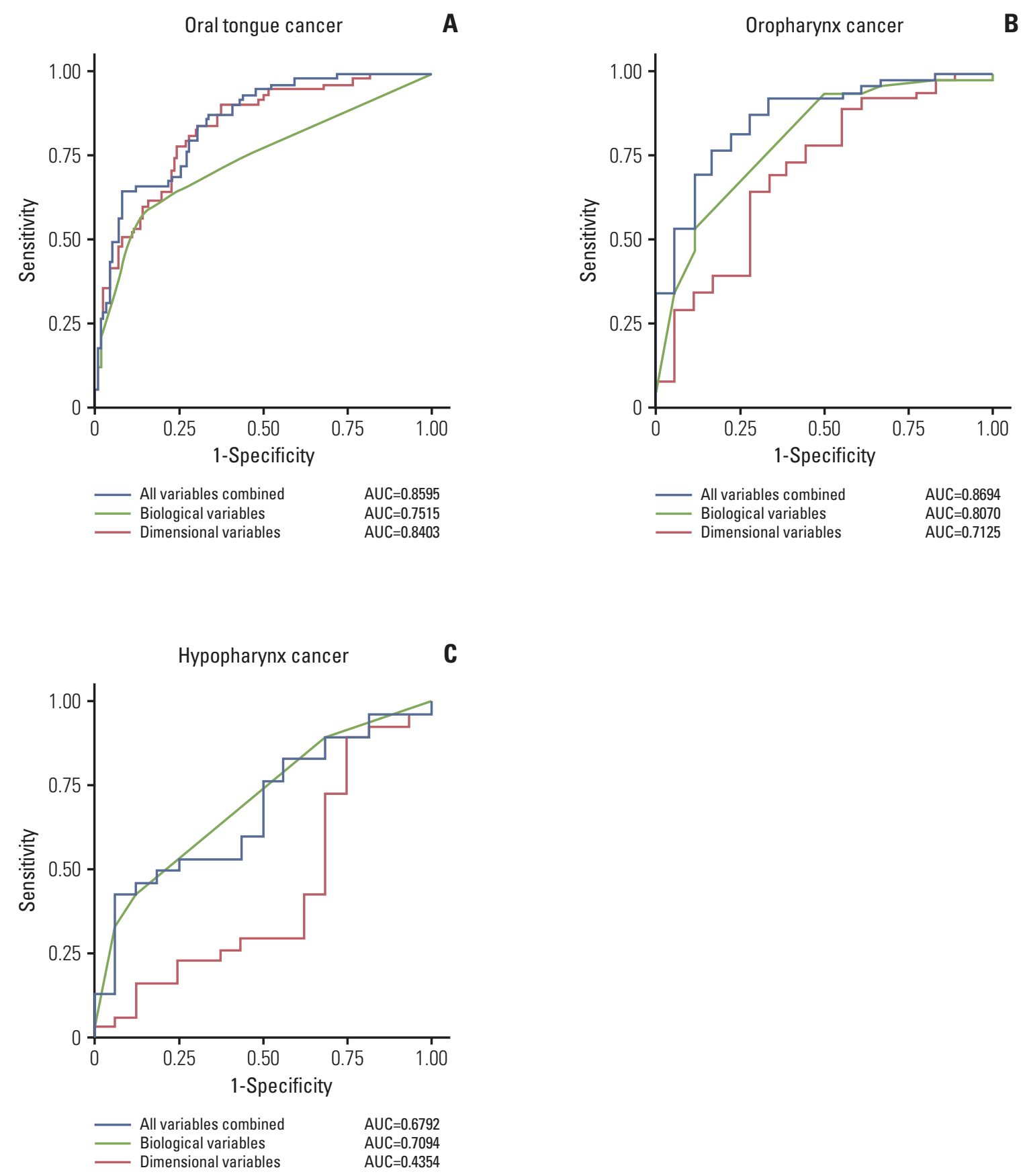

Fig. 2. Predictability for lymph node metastasis according to the tumor subsites. Receiver operating characteristics curve from oral tongue cancer (A), oropharynx cancer (B), and hypopharynx cancer (C). AUC, area under the curves.

\section{Discussion}

LNM acts as a very strong prognostic indicator in many solid cancers, as well as HNSCC, which led to investigations of the determinants of nodal metastatic cascade. In this study, a prediction of LNM in HNSCC based on primary tumor characteristics was analyzed using variables that represent tumor dimension and biology. The overall impact of tumor dimension and biology on LNM was similarly 
Table 3. Cox proportional hazard models for survival outcome of HNSCC patients: subsite analyses

\begin{tabular}{|c|c|c|c|}
\hline \multirow{2}{*}{ Variable } & \multicolumn{3}{|c|}{ Multivariate survival analyses $\left(\mathrm{n}=281^{\mathrm{a}}\right)$} \\
\hline & HR & $95 \% \mathrm{CI}$ & p-value \\
\hline \multicolumn{4}{|l|}{ Oral cavity (tongue) $(\mathrm{n}=166)$} \\
\hline Age & 0.989 & $0.948-1.031$ & 0.593 \\
\hline Sex (male/female) & 1.698 & $0.545-5.2891$ & 0.361 \\
\hline Tumor volume & 0.875 & $0.737-1.039$ & 0.129 \\
\hline Tumor thickness & 4.518 & $1.321-15.447$ & 0.016 \\
\hline LVI (yes/no) & 2.079 & $0.649-6.653$ & 0.218 \\
\hline PNI (yes/no) & 0.572 & $0.160-2.053$ & 0.392 \\
\hline Nodal status $(\mathrm{N} 2+3 / \mathrm{N} 1 / \mathrm{N} 0)$ & 16.566 & $2.412-113.772$ & 0.002 \\
\hline Treatments $(\mathrm{OP}+\mathrm{CCRT} / \mathrm{OP}+\mathrm{RT} / \mathrm{OP})$ & 13.527 & $1.126-162.455$ & 0.008 \\
\hline \multicolumn{4}{|l|}{ Oropharynx $(\mathrm{n}=71)$} \\
\hline Age & 1.104 & $1.009-1.207$ & 0.031 \\
\hline Tumor volume & 0.992 & $0.860-1.144$ & 0.911 \\
\hline Tumor thickness & 1.783 & $0.529-6.002$ & 0.351 \\
\hline LVI (yes/no) & 1.622 & $0.461-5.710$ & 0.452 \\
\hline PNI (yes/no) & 3.714 & $0.291-47.476$ & 0.313 \\
\hline Tumor grade (PD+MD / WD) & 2.190 & $0.113-42.486$ & 0.646 \\
\hline Nodal status (N2+3/N1/N0) & 1.997 & $0.232-17.203$ & 0.248 \\
\hline \multicolumn{4}{|l|}{ Hypopharynx $(n=44)$} \\
\hline Age & 0.933 & $0.861-1.010$ & 0.088 \\
\hline Tumor volume & 1.047 & $0.967-1.133$ & 0.256 \\
\hline Tumor thickness & 1.275 & $0.319-5.085$ & 0.731 \\
\hline LVI (yes/no) & 1.585 & $0.372-6.759$ & 0.534 \\
\hline PNI (yes/no) & 20.314 & 2.324-177.564 & 0.007 \\
\hline Tumor grade $(\mathrm{PD}+\mathrm{MD} / \mathrm{WD})$ & 2.451 & $0.122-49.385$ & 0.775 \\
\hline Treatments $(\mathrm{OP}+\mathrm{CCRT} / \mathrm{OP}+\mathrm{RT})$ & 0.554 & $0.089-3.438$ & 0.526 \\
\hline
\end{tabular}

HNSCC, head and neck squamous cell carcinomas; HR, hazards ratio; CI, confidence interval; LVI, lympho-vascular invasion; PNI, perineural invasion; OP, surgery; CCRT, concurrent chemoradiation; RT, radiotherapy; PD, poorly-differentiated; $M D$, moderately differentiated; $\mathrm{WD}$, well-differentiated. ${ }^{\mathrm{a}} \mathrm{n}=14$ : excluded from survival analyses due to the incomplete medical records.

significant, with AUC of 0.7682 and 0.7717 , respectively. Interestingly, the combined measure of the variables strikingly increased the predictive value for LNM with AUC of 0.8315 . These results are comparable with recent data performed in breast cancer, which indicated the impact of tumor dimension and biology on LNM, with an AUC 0.6700 and 0.6852, respectively [11]. However, the overall predictive value was significantly high in this study in a comparison between HNSCC and those in breast cancer (AUC, $0.8315 \mathrm{vs.}$ 0.7462). These results reflect important characteristics of HNSCC, in which there exist a strong association between primary tumor and metastatic lymph node.

Generally, the dimension of a tumor has been considered to be a product of tumor size. However, the maximal tumor dimension, which is currently used in TNM staging, represents just one-dimensional information. Recently, the total tumor volume as a three-dimensional parameter has been proposed as an important prognostic factor in HNSCC $[14,15]$. Therefore, we adopted an estimated tumor volume based on the pathological reports for three-dimensional analyses. Moreover, tumor thickness is a particularly strong independent predictive factor for LNM in the majority of HNSCC cases $[10,16]$. Hence, we performed a combined measure of estimated tumor volume and tumor thickness as the dimensional variables, which showed a significant and context-dependent impact on LNM in HNSCC.

Tumor biological factors also significantly affect LNM in HNSCC. In this study, the impact of primary tumor biological factors on LNM was determined by a combined measure of LVI, PNI, and tumor grade, which showed a significant correlation with LNM in HNSCC [12]. As expected, LVI, PNI, and tumor grade had significant and synergistic roles on the 
prediction of LNM with AUC of 0.6666, 0.5342, and 0.6518, respectively. In addition, a recent investigation also suggested that several molecular markers, including epidermal growth factor receptor (EGFR) expression, TP53 mutation, and human papillomavirus (HPV) infection status, are emerging prognostic factors and therapeutic targets in HNSCC $[17,18]$. HPV-positive tumors have a relatively favorable prognosis, while tumors with TP53 mutations or EGFR over-expression have a relatively poor prognosis $[19,20]$. Thus, further analyses for these markers as biological factors might give rise to an increase of the predictive value for LNM.

One may argue that our statistical models oversimplified the primary tumor characteristics, just divided into two arbitrary categories: tumor dimension and biology. Indeed, the tumor dimension, such as tumor volume and thickness, are possibly affected by tumor infiltrative activity or tumordoubling time as biological phenomena. Tumor biological factors are also partly reflected by dimensional progression of primary tumor. Thus, we cautiously selected and divided the indicated variables into a more probable side of the categories based on a previous report [11]. Despite the concerns, our simplified model has benefits, providing an acceptable understanding of nodal metastatic cascade and translating easily into the clinics for a more accurate prediction of LNM in different subsites of HNSCC.

One of the intriguing aspects of this study is the tumor subsite-specific analyses of HNSCC, which showed subsitedependent different predictive value of the indicated variables. In fact, each primary tumor site of HNSCC, such as oral tongue cancer and hypopharynx cancer, have been known to have different clinical characteristics, treatment protocols, and prognosis [6]. Concomitantly, we tried to analyze the impact of tumor dimension and biology on LNM in each subsite of HNSCC and showed the context-dependent impact of these variables by different subsites. In oral tongue cancers, a surprisingly high predictability of tumor dimension on LNM with AUC of 0.8403 indicated why local control, through either surgery or radiation therapy, is important [16,21]. Meanwhile, in oropharynx and hypopharynx cancers, a more dominant impact of tumor biological factors and relatively low predictability of tumor dimensional factors on LNM suggest that understanding and therapeutic control of the tumor biology is necessary [18].

Considering LNM in HNSCC as a strong prognostic factor that influences distant metastasis and survival, we tried to confirm our findings through the survival analyses. When we analyzed disease-free (recurrence or distant metastasis) survivals, we could observe a similar trend in survival analyses; tumor dimension (thickness) had a significant prognostic impact on disease-free survival in oral tongue cancer, as biological factor (PNI) did in hypopharynx cancer. However, it is interesting that the variables of primary tumor dimension or biology did not significantly impact the overall survival, despite the impact on LNM in our series. We cautiously interpreted these results to indicate that many host factors, which were not included in the analysis or biological factors in metastatic sites, may have a more direct impact on overall survival. Recently, it has been suggested that the biology and phenotype of primary tumors differ from those of metastatic lymph nodes [22,23], and tumor cells in different microenvironments respond differently to therapy $[22,24]$. Possibly, metastatic nodal characteristics can be independent prognostic factors for distant metastasis and survival in HNSCC [25]. Thus, these points remain to be clarified through the further studies.

\section{Conclusion}

Both dimension and biology of primary tumors have a significant, tumor subsite-dependent impact on the occurrence of LNM in HNSCC. Particularly in oro- and hypo-pharyngeal cancers, tumor biological properties, which can be estimated through a biopsy or surgical pathology of primary tumors, could guide adjuvant treatment for regional lymph nodes. Meanwhile, the primary tumor dimension should be considered in decision making of neck management in oral tongue cancer; as such, an accurate evaluation of tumor dimension is important.

\section{Conflicts of Interest}

Conflict of interest relevant to this article was not reported.

\section{Acknowledgments}

This study was supported by NRF-2012R1A1A2040866 (MEST, South Korea) and Samsung Biomedical Research Institute Grant \#GL1B22912 (South Korea). 


\section{References}

1. Edge SB, Compton CC. The American Joint Committee on Cancer: the 7th edition of the AJCC cancer staging manual and the future of TNM. Ann Surg Oncol. 2010;17:1471-4.

2. Pantel K, Brakenhoff RH. Dissecting the metastatic cascade. Nat Rev Cancer. 2004;4:448-56.

3. Leemans CR, Tiwari R, Nauta JJ, van der Waal I, Snow GB. Regional lymph node involvement and its significance in the development of distant metastases in head and neck carcinoma. Cancer. 1993;71:452-6.

4. Cerezo L, Millan I, Torre A, Aragon G, Otero J. Prognostic factors for survival and tumor control in cervical lymph node metastases from head and neck cancer: a multivariate study of 492 cases. Cancer. 1992;69:1224-34.

5. Le Tourneau C, Velten M, Jung GM, Bronner G, Flesch H, Borel C. Prognostic indicators for survival in head and neck squamous cell carcinomas: analysis of a series of 621 cases. Head Neck. 2005;27:801-8.

6. Argiris A, Karamouzis MV, Raben D, Ferris RL. Head and neck cancer. Lancet. 2008;371:1695-709.

7. Shah JP, Candela FC, Poddar AK. The patterns of cervical lymph node metastases from squamous carcinoma of the oral cavity. Cancer. 1990;66:109-13.

8. Goerkem M, Braun J, Stoeckli SJ. Evaluation of clinical and histomorphological parameters as potential predictors of occult metastases in sentinel lymph nodes of early squamous cell carcinoma of the oral cavity. Ann Surg Oncol. 2010;17: 527-35.

9. Moore C, Flynn MB, Greenberg RA. Evaluation of size in prognosis of oral cancer. Cancer. 1986;58:158-62.

10. Tomifuji M, Imanishi Y, Araki K, Yamashita T, Yamamoto S, Kameyama K, et al. Tumor depth as a predictor of lymph node metastasis of supraglottic and hypopharyngeal cancers. Ann Surg Oncol. 2011;18:490-6.

11. Smeets A, Ryckx A, Belmans A, Wildiers H, Neven P, Floris $\mathrm{G}$, et al. Impact of tumor chronology and tumor biology on lymph node metastasis in breast cancer. Springerplus. 2013; 2:480.

12. Chen TC, Wang CP, Ko JY, Yang TL, Hsu CW, Yeh KA, et al. The impact of perineural invasion and/or lymphovascular invasion on the survival of early-stage oral squamous cell carcinoma patients. Ann Surg Oncol. 2013;20:2388-95.

13. Jones HB, Sykes A, Bayman N, Sloan P, Swindell R, Patel M, et al. The impact of lymphovascular invasion on survival in oral carcinoma. Oral Oncol. 2009;45:10-5.

14. Knegjens JL, Hauptmann M, Pameijer FA, Balm AJ, Hoebers FJ, de Bois JA, et al. Tumor volume as prognostic factor in chemoradiation for advanced head and neck cancer. Head Neck. 2011;33:375-82.
15. Strongin A, Yovino S, Taylor R, Wolf J, Cullen K, Zimrin A, et al. Primary tumor volume is an important predictor of clinical outcomes among patients with locally advanced squamous cell cancer of the head and neck treated with definitive chemoradiotherapy. Int J Radiat Oncol Biol Phys. 2012;82: 1823-30.

16. Ganly I, Goldstein D, Carlson DL, Patel SG, O'Sullivan B, Lee $\mathrm{N}$, et al. Long-term regional control and survival in patients with "low-risk," early stage oral tongue cancer managed by partial glossectomy and neck dissection without postoperative radiation: the importance of tumor thickness. Cancer. 2013; 119:1168-76.

17. Haddad RI, Shin DM. Recent advances in head and neck cancer. N Engl J Med. 2008;359:1143-54.

18. Leemans CR, Braakhuis BJ, Brakenhoff RH. The molecular biology of head and neck cancer. Nat Rev Cancer. 2011;11: 9-22.

19. Ang KK, Berkey BA, Tu X, Zhang HZ, Katz R, Hammond EH, et al. Impact of epidermal growth factor receptor expression on survival and pattern of relapse in patients with advanced head and neck carcinoma. Cancer Res. 2002;62:7350-6.

20. Marur S, D'Souza G, Westra WH, Forastiere AA. HPV-associated head and neck cancer: a virus-related cancer epidemic. Lancet Oncol. 2010;11:781-9.

21. Ganly I, Patel S, Shah J. Early stage squamous cell cancer of the oral tongue--clinicopathologic features affecting outcome. Cancer. 2012;118:101-11.

22. Akita H, Doki Y, Yano M, Miyata H, Miyashiro I, Ohigashi H, et al. Effects of neoadjuvant chemotherapy on primary tumor and lymph node metastasis in esophageal squamous cell carcinoma: additive association with prognosis. Dis Esophagus. 2009;22:291-7.

23. Fruhwirth GO, Diocou S, Blower PJ, Ng T, Mullen GE. A whole-body dual-modality radionuclide optical strategy for preclinical imaging of metastasis and heterogeneous treatment response in different microenvironments. J Nucl Med. 2014; 55:686-94.

24. Padera TP, Kuo AH, Hoshida T, Liao S, Lobo J, Kozak KR, et al. Differential response of primary tumor versus lymphatic metastasis to VEGFR-2 and VEGFR-3 kinase inhibitors cediranib and vandetanib. Mol Cancer Ther. 2008;7:2272-9.

25. Bernier J, Cooper JS, Pajak TF, van Glabbeke M, Bourhis J, Forastiere A, et al. Defining risk levels in locally advanced head and neck cancers: a comparative analysis of concurrent postoperative radiation plus chemotherapy trials of the EORTC (\#22931) and RTOG (\# 9501). Head Neck. 2005;27: 843-50. 